\title{
A General Architecture for Reliable Decentralized Supervisory Control of Discrete Event Systems
}

\author{
Fuchun Liu and Hai Lin
}

\begin{abstract}
In this paper, we study the reliable decentralized supervisory control of discrete event systems (DESs) under the general architecture, in which the decision for controllable events employed is a combination of the conjunctive fusion and disjunctive fusion rules. For a plant equipped with $n$ local supervisors, the notion of $k$-reliable $(1 \leq k \leq n)$ decentralized supervisor is formalized and investigated here. By $k$-reliable decentralized supervisor, we mean that the specification can be achieved exactly even under possible failures of any no more than $n-k$ local supervisors. It is worth noting that the standard decentralized supervisory control problem in the general architecture [14] can be regarded as a special case of $k$-reliable decentralized supervisory control with $k=n$. The main contributions of the paper lie on the proposed necessary and sufficient conditions for the existences of $k$ reliable decentralized supervisor and nonblocking $k$-reliable decentralized supervisor in the context of the general architecture, based on the notions of the $\widetilde{\Sigma}_{u c}$-controllability and $k$-reliably $\widetilde{\Sigma}_{c}$-coobservability of a sublanguage. This represents a generalization of the results in [11].
\end{abstract}

Keywords: Discrete event systems, supervisory control, reliable decentralized supervisor, conjunctive architecture, disjunctive architecture.

\section{INTRODUCTION}

When a system considered is physically distributed, the decentralized supervisory control is commonly regarded as more efficient than the centralized one [9], in which there are a set of local supervisors, each makes control decisions based only on its own direct observations. The past decade has seen increasing research activities in the area of decentralized supervisory control of discrete event systems (DESs), such as [1], [3], [4], [7]-[10], [11]-[15]. In particular, Yoo and Lafortune [14] presented a general architecture for decentralized supervisory control of DESs based on conjunctive and disjunctive fusion rules for local decisions.

Since the general decentralized architecture was initiated by Yoo and Lafortune [14], it has been extensively adopted in the literature. Rohloff and Lafortune [9] presented a new approach of state estimation and safe controllers synthesis in supervisory control of DESs under the general architecture. For the framework of [14], Park and Cho proposed the

This work was supported by the National Natural Science Foundation of China (60974019), Natural Science Foundation of Guangdong Province (9451009001002686) of China, and Singapore Ministry of Education's AcRF Tier 1 funding, TDSI, TL.

F. Liu is with Faculty of Computer, Guangdong University of Technology, Guangzhou 510006, China; and also with Department of Electrical and Computer Engineering, National University of Singapore, 117576, Singapore liufch8@gmail.com

$\mathrm{H}$. Lin is with Department of Electrical and Computer Engineering, National University of Singapore, 117576, Singapore elelh@nus.edu.sg existence condition of a decentralized supervisor for an uncertain DES modeled by a set of possible nondeterministic automata with internal events [8]. Kumar and Takai [3] presented an inference-based ambiguity management in decentralized decision-making in the general decentralized architecture. Reference [15] generalized the architecture of [14] to a conditional architecture, and studied the supervisor existence of decentralized supervisory control with conditional decisions. Park and Cho investigated the decentralized supervisory control of DESs with communication delays based on conjunctive and permissive decision structures [7].

Recently, the reliable decentralized supervisory control of DESs has been formulated in [11] [12]. For a system controlled by $n$ local supervisors, a $k$-reliable $(1 \leq k \leq n)$ decentralized supervisor requires that it exactly achieves the given specification under possible failures of any no more than $n-k$ local supervisors. The authors in [11] presented the conditions for the existence of a $k$-reliable decentralized supervisor by means of the modified controllability and reliable coobservability. It is worth noting that the standard decentralized supervisory control problem [2] can be regarded as a special case with $k=n$ (i.e., synthesizing a $n$-reliable decentralized supervisor).

However, the decentralized architecture considered in [11] [12] is the conjunctive architecture. In this paper, we investigate the following reliable decentralized supervisory control of DESs in the general architecture:

For given a system controlled by $n$ local supervisors and a desired specification, check whether there exists a $k$-reliable decentralized supervisor in the general architecture. If there exists such decentralized supervisor, how to design the local supervisors such that the decentralized supervisor synthesized is $k$-reliable $(1 \leq k \leq n)$.

Firstly, we formalize the notion of $k$-reliable decentralized supervisor in the general architecture. Roughly speaking, a decentralized supervisor is said to be $k$-reliable if the local supervisors achieve exactly the specification under possible failures of any no more than $n-k$ local supervisors, where the decisions for disabled and enabled events are based on the conjunctive and disjunctive fusion rules. Then the concepts of $\widetilde{\Sigma}_{u c}$-controllability and $k$-reliably $\widetilde{\Sigma}_{c}$-coobservability of a language are introduced. In particular, we present the necessary and sufficient conditions for the existence of a $k$-reliable decentralized supervisor in the general architecture by means of the $\widetilde{\Sigma}_{u c}$-controllability and $k$-reliably $\widetilde{\Sigma}_{c^{-}}$coobservability. In addition, we formulate the construction of 
the local supervisors and the reliable decentralized supervisor based on the conjunctive and disjunctive fusion rules.

The approach proposed in this paper is different from those in the literature. The framework of [11] is based on the conjunctive fusion rule for the decisions of disabled events. Reference [12] extends the work of [11] to the case of the marked language specification, which is still in the conjunctive architecture. The architecture adopted by this paper is the general decentralized architecture proposed by Yoo and Lafortune [14]. The notion of $k$-reliable decentralized supervisor and the concepts of $\widetilde{\Sigma}_{u c}$-controllability and $k$-reliably $\widetilde{\Sigma}_{c}$-coobservability introduced in this paper are different from those in [13]. As a result, the local supervisors and the decentralized supervisor constructed in this paper differ from those of [13].

The rest of the paper is organized as follows. Section II recalls some preliminaries of DESs. In Section III, we propose an approach to synthesize a part of local supervisors which is used to deduce the necessary and sufficient conditions of the existence of reliable decentralized supervisors. In Section IV, we investigate the reliable decentralized supervisor in the general architecture. In particular, we present the necessary and sufficient conditions for the existence of a (nonblocking) $k$-reliable decentralized supervisor. In order to illustrate the results proposed, an example is provided in Section V. Finally, in Section VI, we summarize the main results of the paper and address some related issues.

\section{Preliminaries}

Consider a DES modeled by an automaton

$$
G=\left(Q, \Sigma, \delta, q_{0}, Q_{m}\right),
$$

where $Q$ is the set of states, $\Sigma$ is the finite set of events, $\delta: Q \times \Sigma \rightarrow Q$ is the transition function, $q_{0} \in Q$ is the initial state, and $Q_{m} \subseteq Q$ is the set of marked states. Let $\Sigma^{*}$ denote the set of all finite strings over $\Sigma$, including the empty string $\epsilon$. The transition function $\delta$ can be extended to domain $Q \times \Sigma^{*}$ in the following recursive manner: $\delta(q, \epsilon)=q$ and $\delta(q, s \sigma)=\delta(\delta(q, s), \sigma)$ for all $s \in \Sigma^{*}$ and $\sigma \in \Sigma$.

A subset of $\Sigma^{*}$ is called a language. The language generated by $G$, denoted by $L(G)$, is defined by

$$
L(G)=\left\{s \in \Sigma^{*}: \delta\left(q_{0}, s\right) \text { is defined }\right\},
$$

and the language marked by $G$ is defined as

$$
L_{m}(G)=\left\{s \in L(G): \delta\left(q_{0}, s\right) \in Q_{m}\right\} .
$$

For a language $K \subseteq \Sigma^{*}$, we denote the set of all prefixes of strings in $K$ as $\bar{K}$, i.e.,

$$
\bar{K}=\left\{s \in \Sigma^{*}: s t \in K \text { for some } t \in \Sigma^{*}\right\} .
$$

$K$ is called to be prefix-closed if $K=\bar{K}$; and $K$ is called to be $L_{m}(G)$-closed if $K=\bar{K} \cap L_{m}(G)$.

In the decentralized control architecture [2], a system $G$ is jointly controlled by $n$ local supervisors $S_{P_{1}}, S_{P_{2}}, \cdots, S_{P_{n}}$ according to the fusion rule on the local decision actions, and each local supervisor can observe the locally observable events and can control the controllable events. Denote $\Sigma_{i, c}$ and $\Sigma_{i, u c}$ as the sets of locally controllable and uncontrollable events, respectively; $\Sigma_{i, o}$ and $\Sigma_{i, u o}$ as the sets of locally observable and unobservable events, respectively, where $i \in I=\{1,2, \cdots, n\}$. The projection $P_{i}: \Sigma^{*} \rightarrow \Sigma_{i, o}^{*}$ is defined inductively as $P_{i}(\epsilon)=\epsilon$, and for $\sigma \in \Sigma$ and $s \in \Sigma^{*}$,

$$
P_{i}(s \sigma)= \begin{cases}P_{i}(s) \sigma, & \text { if } \sigma \in \Sigma_{i, o} \\ P_{i}(s), & \text { otherwise }\end{cases}
$$

The sets of globally controllable and observable events are respectively defined as

$$
\Sigma_{c}=\bigcup_{i \in I} \Sigma_{i, c}, \quad \Sigma_{o}=\bigcup_{i \in I} \Sigma_{i, o},
$$

and the sets of globally uncontrollable and unobservable events are defined respectively as $\Sigma_{u c}=\Sigma-\Sigma_{c}$ and $\Sigma_{u o}=\Sigma-\Sigma_{o}$.

In this paper, we consider the reliable decentralized supervisory control problem based on the general architecture proposed by Yoo and Lafortune [14], which can be depicted as Fig.1. In the setting of general decentralized control architecture [14], the local supervisors make local enable decision and local disable decision, and the decision fusion for global enable and disable events is a fixed combination of the conjunctive and disjunctive fusions. Formally, the set of controllable events $\Sigma_{c}$ is further partitioned into $\Sigma_{c, e}$ and $\Sigma_{c, d}$, i.e., $\Sigma_{c}=\Sigma_{c, e} \cup \dot{\cup} \Sigma_{c, d}$, where the default setting for controllable events in $\Sigma_{c, e}$ is "enablement" and the default setting for controllable events in $\Sigma_{c, d}$ is "disablement". The local decisions over $\Sigma_{c, e}$ are processed by the conjunctive fusion rule while the local decisions over $\Sigma_{c, d}$ are processed by the disjunctive fusion rule. In addition, we denote $\Sigma_{i, c, e}=$ $\Sigma_{i, c} \cap \Sigma_{c, e}$ and $\Sigma_{i, c, d}=\Sigma_{i, c} \cap \Sigma_{c, d}$.

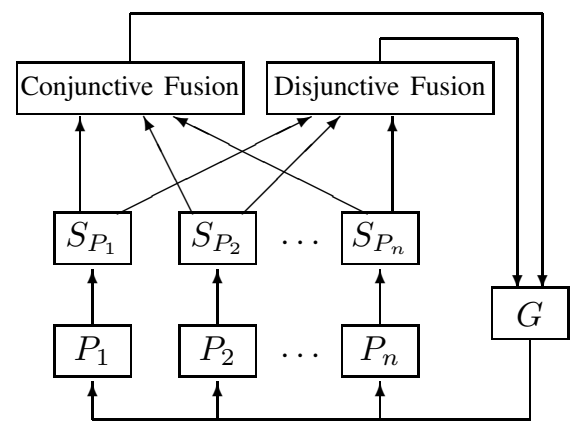

Fig. 1. The general decentralized control architecture.

\section{AN APPROACH OF SyNTHESIS FOR A PART OF LOCAL SUPERVISORS IN GENERAL ARCHITECTURE}

In order to illustrate the reliable decentralized supervisory control of DESs in the general architecture, in this section, we present an approach to synthesize a part of local supervisors based on the conjunctive and disjunctive fusion rules, and then investigate some main properties of the synthesis, which will be used to deduce the conditions of the existence of reliable decentralized supervisor. 
For $\sigma \in \Sigma_{c}$, denote

$$
\operatorname{In}(\sigma)=\left\{i \in I: \sigma \in \Sigma_{i, c}\right\},
$$

where $I=\{1,2, \cdots, n\}$. Let $A \in 2^{I}$, define $\Sigma_{A, c}=$ $\cup_{i \in A} \Sigma_{i, c}$ and $\Sigma_{A, u c}=\Sigma-\Sigma_{A, c}$. For $i \in I$, the local supervisor is defined as a function $S_{P_{i}}: P_{i}\left(\Sigma^{*}\right) \rightarrow \Gamma$, where

$\Gamma=\left\{\gamma \in 2^{\Sigma}: \Sigma_{u c} \cup\left(\Sigma_{c, e}-\Sigma_{i, c}\right) \subseteq \gamma,\left(\Sigma_{c, d}-\Sigma_{i, c}\right) \cap \gamma=\emptyset\right\}$.

Usually, only the observable events can be observed by the local supervisors. Therefore, the local supervisors are supposed to make the same decision for the behaviors with the same projection. This property is formally described as follows.

Definition 1: Let $P_{i}$ be a projection. The local supervisor $S_{P_{i}}$ is called to be feasible if $S_{P_{i}}\left(P_{i}(s)\right)=S_{P_{i}}\left(P_{i}\left(s^{\prime}\right)\right)$ holds for any $s, s^{\prime} \in \Sigma^{*}$ with $P_{i}(s)=P_{i}\left(s^{\prime}\right)$.

Definition 2: For $A \in 2^{I}$, the $A$-decentralized supervisor in the general architecture, denoted by $S_{A}$, is defined as

$$
\begin{aligned}
S_{A}(s)= & P_{\Sigma_{c, e}}\left(\bigcap_{i \in A} S_{P_{i}}\left(P_{i}(s)\right)\right) \\
& \cup P_{\Sigma_{c, d}}\left(\bigcup_{i \in A} S_{P_{i}}\left(P_{i}(s)\right)\right) \cup \Sigma_{A, u c},
\end{aligned}
$$

where $P_{\Sigma_{c, e}}: \Sigma \rightarrow \Sigma_{c, e}$ and $P_{\Sigma_{c, d}}: \Sigma \rightarrow \Sigma_{c, d}$ are projection mappings [14].

Definition 3: The language generated by $S_{A}$, denoted by $L\left(G, S_{A}\right)$, is defined recursively in the usual manner: $\epsilon \in$ $L\left(G, S_{A}\right)$ and for any $s \in \Sigma^{*}$ and $\sigma \in \Sigma$,

$$
s \sigma \in L\left(G, S_{A}\right) \Leftrightarrow s \in L\left(G, S_{A}\right), s \sigma \in L(G), \sigma \in S_{A}(s) .
$$

The marked language $L_{m}\left(G, S_{A}\right)=L\left(G, S_{A}\right) \cap L_{m}(G)$.

Proposition 1: Let $A, B \in 2^{I}$. For language $K \subseteq L(G)$, if $L\left(G, S_{A}\right)=\bar{K}=L\left(G, S_{B}\right)$, then $L\left(G, S_{A \cup B}\right)=\bar{K}$.

Proof: It can be verified directly by induction on the length of the strings in $L\left(G, S_{A}\right), L\left(G, S_{B}\right)$ and $\bar{K}$.

Next, we present the conditions of the existence of $A$ decentralized supervisor $S_{A}$ satisfying $L\left(G, S_{A}\right)=\bar{K}$ by introducing the following notions of the controllability and coobservability of $K$.

Definition 4: Let $A \in 2^{I}$. A language $K \subseteq L(G)$ is said to be $\Sigma_{A, u c}$-controllable if $\bar{K} \Sigma_{A, u c} \cap L(G) \subseteq \bar{K}$.

Definition 5: Let $A \in 2^{I}$. A language $K \subseteq L(G)$ is said to be $\Sigma_{A, c}$-coobservable in the general architecture, if for any $s \in \bar{K}$ and any $\sigma \in \Sigma_{A, c}$, the following conditions hold:

(C1) If $\sigma \in \Sigma_{c, e}$, then $s \sigma \in L(G)-\bar{K}$ implies

$$
(\exists i \in A \cap \operatorname{In}(\sigma)) P_{i}^{-1} P_{i}(s) \sigma \cap \bar{K}=\emptyset ;
$$

(C2) If $\sigma \in \Sigma_{c, d}$, then $s \sigma \in \bar{K}$ implies

$$
(\exists i \in A \cap \operatorname{In}(\sigma))\left(P_{i}^{-1} P_{i}(s) \cap \bar{K}\right) \sigma \cap L(G) \subseteq \bar{K} .
$$

Remark 1: The definition of coobservability under the conjunctive architecture is introduced by Barrett [1] and Cassandras and Lafortune [2], in which language $K$ is called to be coobservable, if for any $s \in \bar{K}$ and any $\sigma \in \Sigma_{c}$ that $s \sigma \in L(G)-\bar{K}$, then there is $i \in I$ such that $\sigma \in \Sigma_{i, c}$ and $P_{i}^{-1} P_{i}(s) \sigma \cap \bar{K}=\emptyset$. For the disjunctive architecture, the notion of coobservability of $K$ in [14] is defined as: for any $s \in \bar{K}$ and any $\sigma \in \Sigma_{c}$ that $s \sigma \in \bar{K}$, then there is $i \in I$ such that $\sigma \in \Sigma_{i, c}$ and $\left(P_{i}^{-1} P_{i}(s) \cap \bar{K}\right) \sigma \cap L(G) \subseteq \bar{K}$. Therefore, when $A=I$ and $\Sigma_{c}=\Sigma_{c, e}$, the above Definition 5 in the general architecture degenerates into the coobservability in the conjunctive architecture; and if $A=I$ and $\Sigma_{c}=\Sigma_{c, d}$, then Definition 5 is consistent with the coobservability in the disjunctive architecture.

Now we present a necessary and sufficient condition for the existence of $A$-decentralized supervisor by using the notions of $\Sigma_{A, u c}$-controllability and $\Sigma_{A, c}$-coobservability.

Theorem 1: Let $A \in 2^{I}$. For a nonempty language $K \subseteq$ $L(G)$, there is an $A$-decentralized supervisor $S_{A}$ such that $L\left(G, S_{A}\right)=\bar{K}$ if and only if $K$ is $\Sigma_{A, u c}$-controllable and $\Sigma_{A, c}$-coobservable.

Proof: $(\Rightarrow)$ For any $s \in \bar{K}$ and any $\sigma \in \Sigma_{A, u c}$ that $s \sigma \in$ $L(G)$, by Eq. (7) and $L\left(G, S_{A}\right)=\bar{K}$, we have $s \in L\left(G, S_{A}\right)$ and $\sigma \in S_{A}(s)$. According to the definition of $L\left(G, S_{A}\right)$, $s \sigma \in L\left(G, S_{A}\right)$, i.e., $s \sigma \in \bar{K}$. Therefore, $\bar{K} \Sigma_{A, u c} \cap L(G) \subseteq$ $\bar{K}$, that is, $K$ is $\Sigma_{A, u c}$-controllable.

Next, we further verify that $K$ is $\Sigma_{A, c}$-coobservable from the following two cases.

Case 1.1: For any $s \in \bar{K}$ and any $\sigma \in \Sigma_{A, c}$, if $\sigma \in \Sigma_{c, e}$ and $s \sigma \in L(G)-\bar{K}$, then $\sigma \notin S_{A}(s)$ for $L\left(G, S_{A}\right)=\bar{K}$. That is, there is $i_{0} \in A$ such that $\left.\sigma \notin S_{P_{i_{0}}}\left(P_{i_{0}}(s)\right)\right)$. From Eq. (6), $\sigma \notin \Sigma_{c, e}-\Sigma_{i_{0}, c}$, i.e., $i_{0} \in \operatorname{In}(\sigma)$. Moreover, if $P_{i_{0}}^{-1} P_{i_{0}}(s) \sigma \cap \bar{K} \neq \emptyset$, then there exists $s^{\prime} \in \Sigma^{*}$ such that $P_{i_{0}}\left(s^{\prime}\right)=P_{i_{0}}(s)$ and $s^{\prime} \sigma \in \bar{K}$. From $L\left(G, S_{A}\right)=\bar{K}$, we have $s^{\prime} \sigma \in L\left(G, S_{A}\right)$, which indicates that $\sigma \in S_{A}\left(s^{\prime}\right)$. Note that $\sigma \in \Sigma_{c, e}$, we obtain $\sigma \in \cap_{i \in A} S_{P_{i}}\left(P_{i}\left(s^{\prime}\right)\right)$, and then $\sigma \in S_{P_{i_{0}}}\left(P_{i_{0}}\left(s^{\prime}\right)\right)$. Due to $P_{i_{0}}\left(s^{\prime}\right)=P_{i_{0}}(s)$, by the feasibility condition (see Definition 1), $\sigma \in S_{P_{i_{0}}}\left(P_{i_{0}}(s)\right.$ ), which is in contradiction with $\left.\sigma \notin S_{P_{i_{0}}}\left(P_{i_{0}}(s)\right)\right)$. Therefore, $P_{i_{0}}^{-1} P_{i_{0}}(s) \sigma \cap \bar{K}=\emptyset$.

Case 1.2: If $\sigma \in \Sigma_{c, d}$ and $s \sigma \in \bar{K}$, then from $L\left(G, S_{A}\right)=$ $\bar{K}$, we have $s \sigma \in L\left(G, S_{A}\right)$, which implies $\sigma \in S_{A}(s)$. So $\sigma \in \cup_{i \in A} S_{P_{i}}\left(P_{i}(s)\right)$ for $\sigma \in \Sigma_{c, d}$. That is, there is $j_{0} \in A$ such that $\sigma \in S_{P_{j_{0}}}\left(P_{j_{0}}(s)\right)$. Notice that $\left(\Sigma_{c, d}-\Sigma_{j_{0}, c}\right) \cap$ $S_{P_{j_{0}}}\left(P_{j_{0}}(s)\right)=\emptyset$, we have $\sigma \in \Sigma_{j_{0}, c}$, i.e., $j_{0} \in \operatorname{In}(\sigma)$. By the definition of $S_{A}$ and $L\left(G, S_{A}\right)=\bar{K}$, it can be proved directly that $\left(P_{j_{0}}^{-1} P_{j_{0}}(s) \cap \bar{K}\right) \sigma \cap L(G) \subseteq \bar{K}$.

$(\Leftarrow)$ Assume that $K$ is $\Sigma_{A, u c^{-c o n t r o l l a b l e} \text { and } \Sigma_{A, c^{-}}}$ coobservable, where $A \in 2^{I}$. For $s \in \Sigma^{*}$ and each $i \in I$, define the local supervisor $S_{P_{i}}\left(P_{i}(s)\right)$ as follows:

$$
\begin{aligned}
& S_{P_{i}}\left(P_{i}(s)\right) \\
= & \left\{\sigma \in \Sigma_{i, c, d}:\left(P_{i}^{-1} P_{i}(s) \cap \bar{K}\right) \sigma \cap L(G) \subseteq \bar{K}\right\} \\
& \cup\left\{\sigma \in \Sigma_{i, c, e}: P_{i}^{-1} P_{i}(s) \sigma \cap \bar{K} \neq \emptyset\right\} \\
& \cup\left(\Sigma_{c, e}-\Sigma_{i, c}\right) \cup \Sigma_{u c},
\end{aligned}
$$

and the $A$-decentralized supervisor $S_{A}$ is defined as Eq. (7).

In order to prove $L\left(G, S_{A}\right)=\bar{K}$, i.e., $s \in L\left(G, S_{A}\right)$ iff $s \in \bar{K}$ for all $s \in \Sigma^{*}$, we show it by induction on the length $|s|$.

If $|s|=0$, i.e., $s=\epsilon$, the base case holds obviously. Suppose that $s \in L\left(G, S_{A}\right)$ iff $s \in \bar{K}$ for any string $s$ with 
$|s| \leq n$. The following is to prove it for $s \sigma$ where $|s|=n$ and $\sigma \in \Sigma$.

Let $s \sigma \in L\left(G, S_{A}\right)$. By the definition of $L\left(G, S_{A}\right)$ and induction hypothesis, we have $s \in \bar{K}, s \sigma \in L(G)$ and $\sigma \in$ $S_{A}(s)$. We verify $s \sigma \in \bar{K}$ from the following cases.

Case 2.1: If $\sigma \in \Sigma_{A, u c}$, then $s \sigma \in \bar{K}$ because of the $\Sigma_{A, u c}$-controllability of $K$.

Case 2.2: If $\sigma \in \Sigma_{A, c} \cap \Sigma_{c, e}$, then we show $s \sigma \in \bar{K}$ by contradiction. Since $K$ is $\Sigma_{A, c}$-coobservable, if $s \sigma \notin \bar{K}$, then there is $i \in A \cap \operatorname{In}(\sigma)$ such that $P_{i}^{-1} P_{i}(s) \sigma \cap \bar{K}=\emptyset$. From Eq. (11), $\sigma \notin S_{P_{i}}\left(P_{i}(s)\right)$ and then $\sigma \notin \cap_{i \in A} S_{P_{i}}\left(P_{i}(s)\right)$, which is in contradiction with $\sigma \in S_{A}(s)$.

Case 2.3: If $\sigma \in \Sigma_{A, c} \cap \Sigma_{c, d}$, then $\sigma \in S_{A}(s)$, which indicates that $\sigma \in \cup_{i \in A} S_{P_{i}}\left(P_{i}(s)\right)$. As a result, there is $i \in A$ such that $\sigma \in S_{P_{i}}\left(P_{i}(s)\right)$. From Eq. (11), we have $\left(P_{i}^{-1} P_{i}(s) \cap \bar{K}\right) \sigma \cap L(G) \subseteq \bar{K}$. Notice that $s \sigma \in$ $\left(P_{i}^{-1} P_{i}(s) \cap \bar{K}\right) \sigma \cap L(G)$, so $s \sigma \in \bar{K}$.

Conversely, let $s \sigma \in \bar{K}$. We check that $s \sigma \in L\left(G, S_{A}\right)$ from the following cases.

Case 3.1: If $\sigma \in \Sigma_{A, u c}$, then $\sigma \in S_{A}(s)$ from the definition of $S_{A}$, which implies $s \sigma \in L\left(G, S_{A}\right)$ since $s \sigma \in$ $L(G)$ and the induction hypothesis $s \in L\left(G, S_{A}\right)$.

Case 3.2: If $\sigma \in \Sigma_{A, c} \cap \Sigma_{c, e}$, it is not difficult to prove $s \sigma \in L\left(G, S_{A}\right)$ with the similar process of Case 2.2.

Case 3.3: If $\sigma \in \Sigma_{A, c} \cap \Sigma_{c, d}$, then from $s \sigma \in \bar{K}$ and the $\Sigma_{A, c}$-coobservability of $K$, there is $i \in A \cap \operatorname{In}(\sigma)$ such that $\left(P_{i}^{-1} P_{i}(s) \cap \bar{K}\right) \sigma \cap L(G) \subseteq \bar{K}$. According to the definition of $S_{P_{i}}$, i.e., Eq. (11), $\sigma \in S_{P_{i}}\left(P_{i}(s)\right)$ and then $\sigma \in \cup_{i \in A} S_{P_{i}}\left(P_{i}(s)\right)$. Consequently, $\sigma \in S_{A}(s)$. By Definition 3, we have $s \sigma \in L\left(G, S_{A}\right)$.

Definition 6: Let $A \in 2^{I}$. The $A$-decentralized supervisor $S_{A}$ is called to be nonblocking if $\overline{L_{m}\left(G, S_{A}\right)}=L\left(G, S_{A}\right)$.

Theorem 2: Let $A \in 2^{I}$. For a nonempty language $K \subseteq$ $L(G)$, there is a nonblocking $A$-decentralized supervisor $S_{A}$ such that $L\left(G, S_{A}\right)=\bar{K}$ and $L_{m}\left(G, S_{A}\right)=K$ if and only if $K$ is $\Sigma_{A, u c}$-controllable, $\Sigma_{A, c}$-coobservable and $L_{m}(G)$ closed.

Proof: $\quad(\Rightarrow)$ The $\Sigma_{A, u c^{-}}$controllability and $\Sigma_{A, c^{-}}$ coobservability of $K$ have been proved in Theorem 1 . From $L\left(G, S_{A}\right)=\bar{K}$ and $L_{m}\left(G, S_{A}\right)=K$, it is easy to show that $K$ is $L_{m}(G)$-closed since

$$
K=L_{m}\left(G, S_{A}\right)=L\left(G, S_{A}\right) \cap L_{m}(G)=\bar{K} \cap L_{m}(G) .
$$

$(\Leftarrow)$ We define the local supervisors and $A$-decentralized supervisor as Eq. (11) and Eq. (7), respectively. By Theorem 1 , we have $L\left(G, S_{A}\right)=\bar{K}$. On the other hand, since $K$ is $L_{m}(G)$-closed, we have

$K=\bar{K} \cap L_{m}(G)=L\left(G, S_{A}\right) \cap L_{m}(G)=L_{m}\left(G, S_{A}\right)$.

As a result, $\overline{L_{m}\left(G, S_{A}\right)}=\bar{K}=L\left(G, S_{A}\right)$, that is, $S_{A}$ is nonblocking.

\section{Reliable Decentralized Supervisory CONTROL IN GENERAL ARCHITECTURE}

Based on the results presented in Section 3, we are ready to investigate the reliable decentralized supervisor under the general architecture, in which a system $G$ is jointly controlled by $n$ local supervisors $S_{P_{1}}, S_{P_{2}}, \cdots, S_{P_{n}}$ according to a fixed combination of the conjunctive and disjunctive fusions on the local decision actions.

Definition 7: Let $K \subseteq L(G)$ be a nonempty language and $1 \leq k \leq n$. The decentralized supervisor $S_{d e c}$ is said to be $k$-reliable in the general architecture, if $L\left(G, S_{A}\right)=\bar{K}$ for any $A \in 2^{I}$ with $|A| \geq k$, where $|A|$ represents the number of elements of $A$, and $S_{A}$ is the $A$-decentralized supervisor in the general architecture.

For $i \in I$, denote $\widetilde{\Sigma}_{i, u c}=\Sigma-\widetilde{\Sigma}_{i, c}$ and

$$
\widetilde{\Sigma}_{i, c}=\left\{\sigma \in \Sigma_{i, c}:|\operatorname{In}(\sigma)| \geq n-k+1\right\} .
$$

Let $A \in 2^{I}$, define $\widetilde{\Sigma}_{A, c}=\cup_{i \in A} \widetilde{\Sigma}_{i, c}$ and $\widetilde{\Sigma}_{A, u c}=\Sigma-\widetilde{\Sigma}_{A, c}$.

For the sake of simplicity, when $A=I$, we denote $\widetilde{\Sigma}_{c}=$ $\widetilde{\Sigma}_{I, c}, \widetilde{\Sigma}_{u c}=\widetilde{\Sigma}_{I, u c}$, and $S_{d e c}=S_{I}$.

Definition 8: A language $K \subseteq L(G)$ is said to be $\widetilde{\Sigma}_{u c^{-}}$ controllable if $\bar{K} \widetilde{\Sigma}_{u c} \cap L(G) \subseteq \overline{\bar{K}}$.

Definition 9: Let $1 \leq \underset{\widetilde{\Sigma}}{k} \leq n$. A language $K \subseteq L(G)$ is said to be $k$-reliably $\widetilde{\Sigma}_{c}$-coobservable in the general architecture, if $\left|A_{s, \sigma}\right| \geq n-k+1$ for any $s \in \bar{K}$ and any $\sigma \in \widetilde{\Sigma}_{c}$, where $A_{s, \sigma}=A_{s, \sigma, 1} \cup A_{s, \sigma, 2}$, and

$$
\begin{gathered}
A_{s, \sigma, 1}=\left\{i \in \operatorname{In}(\sigma): P_{i}^{-1} P_{i}(s) \sigma \cap \bar{K}=\emptyset\right\}, \\
A_{s, \sigma, 2}=\left\{i \in \operatorname{In}(\sigma):\left(P_{i}^{-1} P_{i}(s) \cap \bar{K}\right) \sigma \cap L(G) \subseteq \bar{K}\right\} .
\end{gathered}
$$

Remark 2: The above definition extends the corresponding notion in [11] to the general architecture. In particular, when $\Sigma_{c}=\Sigma_{c, e}$, it degenerates into the notion of reliable $\left(\widetilde{\Sigma}_{c}, k\right)$-coobservability in the conjunctive architecture [11].

Lemma 1: Let $1 \leq k \leq n$ and $K \subseteq L(G)$. There is a $k$-reliable decentralized supervisor $S_{d e c}$ in the general architecture, if and only if, $K$ is $\Sigma_{A, u c}$-controllable and $\Sigma_{A, c}$-coobservable for any $A \in I_{k}$, where

$$
I_{k}=\left\{A \in 2^{I}:|A|=k\right\} \text {. }
$$

Proof: $(\Rightarrow)$ Assume that there is a $k$-reliable decentralized supervisor $S_{d e c}$, then $L\left(G, S_{A}\right)=\bar{K}$ for any $A \in 2^{I}$ with $|A| \geq k$. So $L\left(G, S_{A}\right)=\bar{K}$ for any $A \in I_{k}$. By Theorem 1, we know that $K$ is $\Sigma_{A, u c}$-controllable and $\Sigma_{A, c^{-}}$ coobservable.

$(\Leftarrow)$ Assume that $K$ is $\Sigma_{A, u c^{-c o n t r o l l a b l e} \text { and } \Sigma_{A, c^{-}}}$ coobservable for any $A \in I_{k}$. We define the local supervisor $S_{P_{i}}\left(P_{i}(s)\right)$ as Eq. (11) and the decentralized supervisor $S_{d e c}$ is the same as Eq. (7) with $A=I$.

Next, we prove that $S_{d e c}$ is $k$-reliable, i.e., $L\left(G, S_{B}\right)=\bar{K}$ for any $B \in 2^{I}$ with $|B| \geq k$. If $|B|=k$, then from the assumption, $K$ is $\Sigma_{B, u c}$-controllable and $\Sigma_{B, c}$-coobservable. By Theorem 1 , we have $L\left(G, S_{B}\right)=\bar{K}$. If $|B|>k$, then there are $B_{1}, B_{2}, \cdots, B_{m}$ such that

$$
B=B_{1} \cup B_{2} \cup \cdots \cup B_{m}
$$

and $B_{i} \in I_{k}$ for each $B_{i}$. By the assumption, $K$ is $\Sigma_{B_{i}, u c^{-}}$ controllable and $\Sigma_{B_{i}, c}$-coobservable for each $B_{i}$. According to Theorem 1, we have

$$
L\left(G, S_{B_{1}}\right)=L\left(G, S_{B_{2}}\right)=\cdots=L\left(G, S_{B_{m}}\right)=\bar{K},
$$


which implies $L\left(G, S_{B}\right)=\bar{K}$ according to Proposition 1 . So $S_{d e c}$ is $k$-reliable.

Lemma 2: Let $1 \leq k \leq n$ and $K \subseteq L(G) . K$ is $\widetilde{\Sigma}_{u c^{-}}$ controllable and $k$-reliably $\widetilde{\Sigma}_{c}$-coobservable, if and only if, $K$ is $\Sigma_{A, u c^{-c o n t r o l l a b l e}}$ and $\Sigma_{A, c^{-}}$-coobservable for any $A \in$ $I_{k}$, where $I_{k}=\left\{A \in 2^{I}:|A|=k\right\}$.

Proof: $(\Leftarrow)$ We first prove that $K$ is $\widetilde{\Sigma}_{u c}$-controllable, i.e., $\bar{K} \widetilde{\Sigma}_{u c} \cap L(G) \subseteq \bar{K}$. Denote

$$
\Sigma_{c}(k)=\left\{\sigma \in \Sigma_{c}:|\operatorname{In}(\sigma)| \leq n-k\right\},
$$

then

$$
\bar{K} \widetilde{\Sigma}_{u c} \cap L(G)=\left(\bar{K} \Sigma_{c}(k) \cap L(G)\right) \cup\left(\bar{K} \Sigma_{u c} \cap L(G)\right) .
$$

For any $s \sigma \in \bar{K} \Sigma_{c}(k) \cap L(G)$, we have $\sigma \in \Sigma_{c}$ and $|\operatorname{In}(\sigma)| \leq n-k$, which shows that there is $B \in I_{k}$ such that $\sigma \in \Sigma_{B, u c}$. Notice that $K$ is $\Sigma_{B, u c}$-controllable, i.e., $\bar{K} \Sigma_{B, u c} \cap L(G) \subseteq \bar{K}$, so $s \sigma \in \bar{K}$. That is, $\bar{K} \Sigma_{c}(k) \cap L(G) \subseteq$ $\bar{K}$. On the other hand, $\bar{K} \Sigma_{u c} \cap L(G) \subseteq \bar{K}$ since

$$
\bar{K} \Sigma_{u c} \cap L(G) \subseteq \bar{K} \Sigma_{B, u c} \cap L(G) .
$$

Therefore, $\bar{K} \widetilde{\Sigma}_{u c} \cap L(G) \subseteq \bar{K}$.

Next, we verify that $K$ is $k$-reliably $\widetilde{\Sigma}_{c}$-coobservable by contradiction. Suppose that there is $s \in \bar{K}$ and $\sigma \in \widetilde{\Sigma}_{c}$ satisfying $\left|A_{s, \sigma}\right| \leq n-k$, then $|\operatorname{In}(\sigma)| \geq n-k+1$ and $\left|\operatorname{In}(\sigma)-A_{s, \sigma}\right| \geq 1$. Therefore, there is $j \in \operatorname{In}(\sigma)-A_{s, \sigma}$ and $B \in I_{k}$ such that $A_{s, \sigma} \cap B=\emptyset$ and $j \in B$, which implies $\sigma \in \Sigma_{B, c}$. Note that $K$ is $\Sigma_{B, c}$-coobservable, for the above $s$ and $\sigma$, if $\sigma \in \Sigma_{c, e}$ and $s \sigma \in L(G)-\bar{K}$, then there exists $\ell \in B$ satisfying $\ell \in \operatorname{In}(\sigma)$ and $P_{\ell}^{-1} P_{\ell}(s) \sigma \cap \bar{K}=\emptyset$, i.e., $\ell \in$ $A_{s, \sigma, 1} \subseteq A_{s, \sigma}$. Hence $\ell \in A_{s, \sigma} \cap B$, which is in contradiction with $A_{s, \sigma} \cap B=\emptyset$. On the other side, if $\sigma \in \Sigma_{c, d}$ and $s \sigma \in \bar{K}$, then there exists $h \in B$ satisfying $h \in \operatorname{In}(\sigma)$ and $\left(P_{h}^{-1} P_{h}(s) \cap \bar{K}\right) \sigma \cap L(G) \subseteq \bar{K}$, i.e., $h \in A_{s, \sigma, 2} \subseteq A_{s, \sigma}$. Hence $h \in A_{s, \sigma} \cap B$, which is also in contradiction with $A_{s, \sigma} \cap B=\emptyset$.

$(\Rightarrow)$ We first prove that $K$ is $\Sigma_{A, u c}$-controllable for any $A \in I_{k}$. Since

$$
\begin{aligned}
\Sigma_{A, u c} & =\Sigma_{u c} \cup\left\{\sigma \in \Sigma_{c}: \sigma \notin \Sigma_{A, c}\right\} \\
& \subseteq \Sigma_{u c} \cup\left\{\sigma \in \Sigma_{c}:|\operatorname{In}(\sigma)| \leq n-k\right\} \\
& =\widetilde{\Sigma}_{u c},
\end{aligned}
$$

where $A \in I_{k}$. Therefore, $\bar{K} \Sigma_{A, u c} \cap L(G) \subseteq \bar{K} \widetilde{\Sigma}_{u c} \cap$ $L(G) \subseteq \bar{K}$ by the $\widetilde{\Sigma}_{u c}$-controllability of $K$.

Next, we prove that $K$ is $\Sigma_{A, c}$-coobservable for any $A \in$ $I_{k}$. For any $s \in \bar{K}$ and any $\sigma \in \Sigma_{A, c}$, the proof is completed by the following two cases.

Case 1: If $\sigma \in \Sigma_{A, c} \cap \widetilde{\Sigma}_{c}$, i.e., $|\operatorname{In}(\sigma)| \geq n-k+1$, then $\left|A_{s, \sigma}\right| \geq n-k+1$ since $K$ is $k$-reliably $\widetilde{\Sigma}_{c}$-coobservable. Notice that $|A|=k$, we have $A \cap A_{s, \sigma} \neq \emptyset$, that is, there is $i \in A$ such that $i \in \operatorname{In}(\sigma)$ and $i \in A_{s, \sigma, 1} \cup A_{s, \sigma, 2}$. When $\sigma \in \Sigma_{c, e}$ and $s \sigma \in L(G)-\bar{K}$, by Eq. (13), it is obtained that $i \notin A_{s, \sigma, 2}$. Therefore, $i \in A_{s, \sigma, 1}$, i.e., $P_{i}^{-1} P_{i}(s) \sigma \cap \bar{K}=\emptyset$. On the other hand, when $\sigma \in \Sigma_{c, d}$ and $s \sigma \in \bar{K}$, by Eq. (12), we have $i \notin A_{s, \sigma, 1}$ for $s \sigma \in P_{i}^{-1} P_{i}(s) \sigma \cap \bar{K}$. So, $i \in A_{s, \sigma, 2}$, i.e., $\left(P_{i}^{-1} P_{i}(s) \cap \bar{K}\right) \sigma \cap L(G) \subseteq \bar{K}$. We complete the proof of $\Sigma_{A, c}$-coobservability of $K$ in the first case.
Case 2: If $\sigma \in \Sigma_{A, c}-\left(\Sigma_{A, c} \cap \widetilde{\Sigma}_{c}\right)$, i.e., $\sigma \in \Sigma_{A, c}$ and $\sigma \in \widetilde{\Sigma}_{u c}$, by the $\widetilde{\Sigma}_{u c}$-controllability of $K$, it can be verified straight that the conditions of Definition 5 hold.

From Lemma 1 and Lemma 2, we have the following necessary and sufficient conditions for the existence of a $k$ reliable decentralized supervisor in the general architecture.

Theorem 3: Let $1 \leq k \leq n$ and $K \subseteq L(G)$. There is a $k$ reliable decentralized supervisor in the general architecture, if and only if, $K$ is $\widetilde{\Sigma}_{u c}$-controllable and $k$-reliably $\widetilde{\Sigma}_{c^{-}}$ coobservable.

Proof: It is a combination of Lemma 1 and Lemma 2.

Remark 3: The existence conditions of Theorem 3 generalize the results of [11] to the general architecture. When $\Sigma_{c}=\Sigma_{c, e}$, Theorem 3 degenerates into the necessary and sufficient conditions of $k$-reliable decentralized supervisor in conjunctive architecture [11].

Theorem 4: Let $1 \leq k \leq n$ and $K \subseteq L(G)$. There is a nonblocking $k$-reliable decentralized supervisor $S_{d e c}$ in the general architecture such that $L_{m}\left(G, S_{A}\right)=K$ for any $A \in 2^{I}$ with $|A| \geq k$, if and only if, $K$ is $\widetilde{\Sigma}_{u c}$-controllable, $k$-reliably $\widetilde{\Sigma}_{c}$-coobservable and $L_{m}(G)$-closed.

Proof: $(\Rightarrow)$ Due to Theorem 3, we only need to prove that $K$ is $L_{m}(G)$-closed. From Definition 7 and $L_{m}\left(G, S_{A}\right)=$ $K$, we have

$K=L_{m}\left(G, S_{A}\right)=L\left(G, S_{A}\right) \cap L_{m}(G)=\bar{K} \cap L_{m}(G)$.

$(\Leftarrow)$ By Theorem 3, there is a $k$-reliable decentralized supervisor $S_{d e c}$ such that $L\left(G, S_{A}\right)=\bar{K}$ for any $A \in 2^{I}$ with $|A| \geq k$. On the other hand, since $K$ is $L_{m}(G)$-closed,

$K=\bar{K} \cap L_{m}(G)=L\left(G, S_{A}\right) \cap L_{m}(G)=L_{m}\left(G, S_{A}\right)$.

Therefore, $K=L_{m}\left(G, S_{d e c}\right)$ and

$$
\overline{L_{m}\left(G, S_{d e c}\right)}=\bar{K}=L\left(G, S_{d e c}\right),
$$

that is, $S_{d e c}$ is nonblocking.

Remark 4: Theorem 4 is a generalization of one of the main results presented in [14]. In fact, the necessary and sufficient conditions for the existence of nonblocking decentralized supervisor in [14] (Theorem 1 on page 343 of [14]) are exactly consistent with those of nonblocking $n$ reliable decentralized supervisor of Theorem 4 (i.e., $k=n$ in Theorem 4).

\section{An Illustrative EXAMPle}

Theorem 3 shows that we can check the existence of the $k$-reliable decentralized supervisor by means of testing the $\widetilde{\Sigma}_{u c}$-controllability and $k$-reliably $\widetilde{\Sigma}_{c}$-coobservability of $K$. Next, an illustrative example is given in this section.

Example 1: Consider a DES $G$ modeled by an automaton shown in Fig. 2. Let $n=3$ (i.e., $I=\{1,2,3\}$ ) and

$$
\begin{array}{lll}
\Sigma_{1, o}=\left\{\sigma_{1}, \sigma_{2}, \sigma_{5}\right\} ; & \Sigma_{1, c, e}=\left\{\sigma_{1}\right\}, & \Sigma_{1, c, d}=\left\{\sigma_{2}\right\} ; \\
\Sigma_{2, o}=\left\{\sigma_{1}, \sigma_{4}\right\} ; & \Sigma_{2, c, e}=\left\{\sigma_{1}\right\}, & \Sigma_{2, c, d}=\left\{\sigma_{4}\right\} ; \\
\Sigma_{3, o}=\left\{\sigma_{2}, \sigma_{3}\right\} ; & \Sigma_{3, c, e}=\left\{\sigma_{3}\right\}, & \Sigma_{3, c, d}=\left\{\sigma_{2}\right\} .
\end{array}
$$




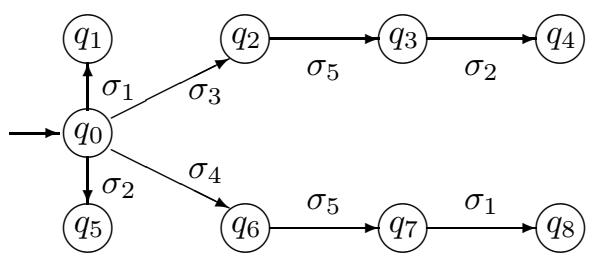

Fig. 2. A DES $G$.

Consider language $K=\overline{\sigma_{1}+\sigma_{2}+\sigma_{4} \sigma_{5}+\sigma_{3} \sigma_{5}}$, then $K \subseteq L(G)$. In the following, we verify that there is a 2 reliable decentralized supervisor by checking that $K$ is $\widetilde{\Sigma}_{u c^{-}}$ controllable and 2-reliably $\widetilde{\Sigma}_{c}$-coobservable, where

$$
\widetilde{\Sigma}_{c}=\left\{\sigma \in \Sigma_{c}:|\operatorname{In}(\sigma)| \geq 2\right\}=\left\{\sigma_{1}, \sigma_{2}\right\},
$$

and $\widetilde{\Sigma}_{u c}=\Sigma-\widetilde{\Sigma}_{c}=\left\{\sigma_{3}, \sigma_{4}, \sigma_{5}\right\}$.

(1) $K$ is $\widetilde{\Sigma}_{u c}$-controllable because

$$
\bar{K} \widetilde{\Sigma}_{u c} \cap L(G)=\left\{\sigma_{3}, \sigma_{4}, \sigma_{3} \sigma_{5}, \sigma_{4} \sigma_{5}\right\} \subseteq \bar{K} .
$$

(2) Next we prove that $K$ is 2-reliably $\widetilde{\Sigma}_{c}$-coobservable, i.e., for any $s \in \bar{K}$ and any $\sigma \in \widetilde{\Sigma}_{c},\left|A_{s, \sigma}\right| \geq 2$ holds. In fact, on the one hand, only $\sigma=\sigma_{1}$ and $s=\sigma_{4} \sigma_{5}$ satisfies $s \in \bar{K}, \sigma \in \widetilde{\Sigma}_{c}, \sigma \in \Sigma_{c, e}$ and $s \sigma \in L(G)-\bar{K}$, and then

$$
A_{s, \sigma, 1}=\left\{i \in \operatorname{In}(\sigma): P_{i}^{-1} P_{i}(s) \sigma \cap \bar{K}=\emptyset\right\}=\{1,2\}
$$

and

$$
A_{s, \sigma, 2}=\left\{i \in \operatorname{In}(\sigma):\left(P_{i}^{-1} P_{i}(s) \cap \bar{K}\right) \sigma \cap L(G) \subseteq \bar{K}\right\}=\emptyset .
$$

Therefore, $\left|A_{s, \sigma}\right|=\left|A_{s, \sigma, 1} \cup A_{s, \sigma, 2}\right|=2$.

On the other hand, only $\sigma=\sigma_{2}$ and $s=\epsilon$ satisfies $s \in \bar{K}$, $\sigma \in \widetilde{\Sigma}_{c}, \sigma \in \Sigma_{c, d}$ and $s \sigma \in \bar{K}$, in this case, we can calculate $A_{s, \sigma, 1}=\emptyset$ and $A_{s, \sigma, 2}=\{1,3\}$. Therefore, $\left|A_{s, \sigma}\right|=2$. By Definition $9, K$ is 2-reliably $\widetilde{\Sigma}_{c}$-coobservable.

Therefore, according to Theorem 3, there is a 2-reliable decentralized supervisor in the general architecture.

In fact, from Eq. (11), the local supervisors can be designed as follows:

$$
\begin{gathered}
S_{P_{1}}\left(P_{1}(s)\right)= \begin{cases}\left\{\sigma_{1}, \sigma_{2}, \sigma_{3}, \sigma_{5}\right\}, & \text { if } P_{1}(s)=\epsilon, \\
\left\{\sigma_{3}, \sigma_{5}\right\}, & \text { if } P_{1}(s)=\sigma_{5}, \\
\left\{\sigma_{2}, \sigma_{3}, \sigma_{5}\right\}, & \text { otherwise. }\end{cases} \\
S_{P_{2}}\left(P_{2}(s)\right)= \begin{cases}\left\{\sigma_{1}, \sigma_{3}, \sigma_{4}, \sigma_{5}\right\}, & \text { if } P_{2}(s)=\epsilon, \\
\left\{\sigma_{3}, \sigma_{4}, \sigma_{5}\right\}, & \text { otherwise. }\end{cases} \\
S_{P_{3}}\left(P_{3}(s)\right)= \begin{cases}\left\{\sigma_{1}, \sigma_{2}, \sigma_{3}, \sigma_{5}\right\}, & \text { if } P_{3}(s)=\epsilon, \\
\left\{\sigma_{1}, \sigma_{5}\right\}, & \text { if } P_{3}(s)=\sigma_{3}, \\
\left\{\sigma_{1}, \sigma_{2}, \sigma_{5}\right\}, & \text { otherwise. }\end{cases}
\end{gathered}
$$

Consequently, we may check straight that the decentralized supervisor is 2-reliable, since

$$
L\left(G, S_{A}\right)=\left\{\epsilon, \sigma_{1}, \sigma_{2}, \sigma_{3}, \sigma_{4}, \sigma_{3} \sigma_{5}, \sigma_{4} \sigma_{5}\right\}=\bar{K}
$$

for any $A \in 2^{I}$ with $|A| \geq 2$.

\section{CONCLUSION}

In this paper, we have investigated the reliable decentralized supervisory control problem without communication based on a combination of the conjunctive and disjunctive fusion rules, which has generalized the results in the conjunctive architecture [11]. Firstly, we have presented an approach to synthesize part of local supervisors, and formulated the notion of $k$-reliable decentralized supervisor in the general architecture. Then the concepts of the $\widetilde{\Sigma}_{u c}$-controllability and $k$-reliably $\widetilde{\Sigma}_{c}$-coobservability of a sublanguage were introduced. Based on these new concepts, necessary and sufficient conditions for the existence of a (nonblocking) $k$-reliable decentralized supervisor were presented in the context of the general architecture.

With the results obtained in this paper, we will consider the reliable robust nonblocking supervisory control of DESs with communication [1] and continue our previous work of [5], [6] to investigate the reliable control for stochastic discrete event systems in the subsequent work.

\section{REFERENCES}

[1] G. Barrett and S. Lafortune, "Decentralized Supervisory Control with Communicating Controllers," IEEE Trans. Automat. Contr., vol. 45, pp. $1620-1638$, Oct. 2000.

[2] C.G. Cassandras and S. Lafortune, Introduction to Discrete Event Systems. Boston, MA: Kluwer, 1999.

[3] R. Kumar and S. Takai, "Inference-Based Ambiguity Management in Decentralized Decision-Making: Decentralized Control of Discrete Event Systems," IEEE Trans. Automat. Contr, vol. 52, no. 10, pp. 1783-1794, 2007.

[4] F. Lin and W.M. Wonham, "Decentralized Control and Coordination of Discrete Event Systems with Partial Observation," IEEE Trans. Automat. Contr., vol. 35, pp. 1330-1337, Dec. 1990.

[5] F.C. Liu, D.W. Qiu, H. Xing, and Z. Fan, "Decentralized Diagnosis of Stochastic Discrete Event Systems," IEEE Trans. Automat. Contr. vol. 53, no. 2, pp. 535-546, 2008.

[6] F.C. Liu and D.W. Qiu, "Safe Diagnosability of Stochastic Discrete Event Systems," IEEE Trans. Automat. Contr., vol. 53, no. 5, pp. 12911296, 2008.

[7] S.J. Park and K.H. Cho, "Decentralized Supervisory Control of Discrete Event Systems with Communication Delays Based on Conjunctive and Permissive Decision Structures," Automatia, vol. 43, pp. 738-743, 2007.

[8] S.J. Park and K. H. Cho, "Decentralized Supervisory Control of Nondeterministic Discrete Event Systems: The Existence Condition of a Robust and Nonblocking Supervisor," Automatica, vol. 43, pp. 377-383, 2007.

[9] K. Rohloff and S. Lafortune, "On the Synthesis of Safe Control Policies in Decentralized Control of Discrete Event Systems," IEEE Trans. Automat. Contr., vol. 48, no. 6, pp. 1064-1068, 2003.

[10] K. Rudie and W. M. Wonham, "Think Globally, Act Locally: Decentralized Supervisory Control," IEEE Trans. Automat. Contr., vol. 37, no. 11, pp. 1692-1708, Nov. 1992.

[11] S. Takai and T. Ushio, "Reliable Decentralized Supervisory Control of Discrete Event Systems," IEEE Trans. Syst., Man, Cubern.-B: Cubern., vol. 30 , no. 5, pp. 661-667, 2000.

[12] S. Takai and T. Ushio, "Reliable Decentralized Supervisory Control for Marked Language Specifications," Asian J. Contr., vol. 5, no. 1, 2003.

[13] S. Takai and T. Ushio, "Reliable Decentralized Supervisory Control of Discrete Event Systems with the Conjunctive and Disjunctive Fusion Rules," in Proc. 2003 Amer. Contr. Conf., June 2003, pp. 1050-1055.

[14] T.-S. Yoo and S. Lafortune, "A General Architecture for Decentralized Supervisory Control of Discrete-Event Systems," Discrete Event Dynamic Systems: Theory and Applications, 12(3), 335-377, 2002.

[15] T.-S. Yoo and S. Lafortune, "Decentralized Supervisory Control with Conditional Decisions: Supervisor Existence," IEEE Trans. Automat. Contr., 49(11), 1886-1904, 2004. 